\title{
Vigilância popular ambiental e siderurgia: as experiências de Piquiá de Baixo (MA) e Santa Cruz (RJ), Brasil
}

\author{
Environmental surveillance of the steel industry by the people: \\ the experiences of Piquiá de Baixo (MA) and Santa Cruz (RJ)
}

Leandro Vargas Barreto de Carvalho (https://orcid.org/0000-0002-3090-7477) ${ }^{1}$

Flávio Rocha (https://orcid.org/0000-0002-2769-8977) ${ }^{2}$

Renan Finamore (https://orcid.org/0000-0002-5835-4771) ${ }^{3}$

${ }^{1}$ Laboratório de Toxicologia. Centro de Estudos da Saúde do Trabalhador e Ecologia Humana, Escola Nacional de Saúde Pública Sergio Arouca, Fundação Oswaldo Cruz. Rua Leopoldo Bulhões, 1480, Manguinhos. 21041-210 Rio de Janeiro RJ Brasil.leandro.carvalho@ ensp.fiocruz.br

${ }^{2}$ Programa de PósGraduação em Ciências Sociais, Universidade do Estado do Rio de Janeiro. Rio de Janeiro RJ Brasil.

${ }^{3}$ Escola Politécnica,

Universidade Federal do Rio

de Janeiro. Rio de Janeiro

RJ Brasil.

\begin{abstract}
Environmental surveillance by the people fosters the protagonism of individuals in a situation of environmental vulnerability to generate and evaluate data regarding the pollution to which they are exposed. The scope of this work was to analyze such experiences in two areas impacted by the steel industry: Santa Cruz/RJ and Piquiá de Baixo/MA. At these sites, community environmental monitoring (CEM) activities were conducted to measure 2.5 micrometer particulate matter (PM2.5) levels in the atmosphere, between November/2016 and July/2017. Such activities were conducted using a low-cost and easy-to-operate air quality monitor. Monthly averages of PM2.5 levels in Piquiá and Santa Cruz were high, depending on the period assessed, since they surpassed the annual average recommended by the World Health Organization (WHO) of $10 \mu \mathrm{g} / \mathrm{m} 3$, and, on several occasions, the recommended daily average of $25 \mu \mathrm{g} / \mathrm{m} 3$. The main idea of this monitoring proposal was to establish a process capable of presenting a counterpoint to official information on local atmospheric pollution scenarios, with the involvement of affected communities. The pollution data produced enable these communities to participate in public debates and decision-making processes in a more informed way.
\end{abstract}

Key words Environmental surveillance by the people, Community environmental monitoring, Air pollution, Particulate matter
Resumo A vigilância popular ambiental (VPA) promove o protagonismo dos sujeitos em situação de vulnerabilidade ambiental na produção e avaliação de dados sobre a poluição a que estão submetidos. $O$ objetivo deste trabalho foi analisar as experiências de VPA desenvolvidas em duas localidades impactadas por siderúrgicas: Santa Cruz/RJ e Piquiá de Baixo/MA. Nesses locais foram realizadas atividades de monitoramento ambiental comunitário (MAC) dos niveis de material particulado de 2,5 micrômetros $\left(\mathrm{MP}_{2,5}\right)$ na atmosfera, entre novembro de 2016 e julho de 2017, utilizando equipamento monitor de qualidade do ar de baixo custo e fácil operação. As médias mensais dos níveis de $M_{2,5}$ em Piquiá e Santa Cruz foram elevadas, a depender da época avaliada, pois ultrapassaram a média anual recomendada pela Organização Mundial de Saúde (OMS), de $10 \mu \mathrm{g} / \mathrm{m}^{3}$, e por várias vezes a média diária recomendada, de $25 \mu \mathrm{g} / \mathrm{m}^{3}$. A ideia principal desta proposta de monitoramento consistiu em estabelecer, com o engajamento das comunidades atingidas, um processo capaz de apresentar um contraponto às informações oficiais sobre cenários locais de poluição atmosférica. Os dados de poluição produzidos possibilitam que essas comunidades tenham uma atuação mais qualificada nos espaços públicos de debate e tomada de decisão.

Palavras-chave Vigilância popular ambiental, Monitoramento ambiental comunitário, Poluição do ar, Material particulado 


\section{Introdução}

A indústria siderúrgica se caracteriza por seu elevado potencial poluidor e de consumo de recursos naturais, como minério de ferro e carvão (vegetal ou mineral). Conforme Souza ${ }^{1}$, a fabricação de aço demanda consideráveis quantidades de água e energia, além de lançar poluentes em corpos hídricos e na atmosfera. Entre as emissões atmosféricas, além de gases de efeito estufa, há diversos poluentes locais, como benzeno, material particulado (MP) e óxido de enxofre, que podem causar problemas de saúde, sendo associados a doenças respiratórias, oftalmológicas, dermatológicas e até mesmo câncer².

As comunidades que habitam a circunvizinhança de empreendimentos siderúrgicos são, em geral, bastante impactadas. E não raro surgem mobilizações sociais críticas à operação dessas instalações industriais. As reivindicações buscam, majoritariamente, reparações por direitos sociais e ambientais violados, além de colocar em questão o modo operatório de tais empreendimentos, que intensificam e criam situações de exposição humana a riscos ambientais e industriais ${ }^{3}$.

No contexto brasileiro, dois exemplos emblemáticos de tais mobilizações são as que ocorrem nas localidades de Santa Cruz, município do Rio de Janeiro (RJ), e Piquiá de Baixo, município de Açailândia (MA). Apesar das particularidades de cada caso, há pelo menos dois pontos em comum: (1) ambos evidenciam situações de injustiça ambiental em que se verifica uma desigualdade na distribuição dos riscos e impactos ambientais gerados pelas atividades produtivas poluidoras, sobrecarregando as comunidades circunvizinhas ${ }^{4}$ (2) as duas comunidades atingidas se articularam para a produção de dados sobre a poluição atmosférica local por MP, a fim de melhorar a compreensão técnica do problema que vivenciam e denunciam há anos.

Deve-se pontuar que as empresas siderúrgicas, como as que estão presentes em Piquiá e Santa Cruz, tendem a lidar com conflitos ambientais que envolvem diferentes grupos, com usos e significados distintos atribuídos a um território, que se inicia quando a apropriação de um deles se encontra ameaçada por impactos indesejáveis ao meio ambiente ${ }^{4}$. Esses conflitos estão propensos a denúncias feitas por grupos afetados, movimentos sociais, universidades ou qualquer outro ator engajado nas causas ambientais.

Os empresários propagam discursos para difundir suas visões para a população e argumentam sobre as vantagens econômicas dos empre- endimentos para o país e para as regiões onde se instalam. O principal argumento é o desenvolvimento local, reforçando sua empregabilidade, seu aquecimento à economia e à infraestrutura. Em geral, empresas como a Ternium (antiga TKCSA), em Santa Cruz, e as que operam no polo siderúrgico de Piquiá se instalam em locais de baixa renda, muitas vezes desprovidos de serviços públicos essenciais, e buscam ofuscar a crítica dos moradores construindo postos de saúde, creches, hortas comunitárias etc., muitas das vezes exercendo o papel que deveria ser do Estado promovendo o que se denomina de geopolítica empresarial de gestão de territórios ${ }^{5}$.

Entretanto, as comunidades ambientalmente impactadas buscam suprir a carência de informações públicas confiáveis que reflitam o quadro percebido e sentido em cada localidade. As informações oficiais disponibilizadas, tanto pelas empresas poluidoras envolvidas como pelos órgãos ambientais de fiscalização, são consideradas insuficientes ou insatisfatórias pelos grupos atingidos. Consequentemente, suas denúncias tendem a ser classificadas como frágeis do ponto de vista da argumentação técnica, o que dificulta o reconhecimento público do problema em questão, assim como a legitimidade das reivindicações por reparações pelos danos sofridos ${ }^{3,6}$.

Uma alternativa encontrada pelos atingidos foi buscar abordagens de pesquisa que os permitissem produzir os próprios dados sobre as concentrações de MP a que estão expostos em seus territórios. Tais estratégias de produção de conhecimento acerca da poluição são adotadas por muitas comunidades em situação de injustiça ambiental, como apontam Porto e Finamo$\mathrm{re}^{6}$. A ideia, portanto, consistiu em estabelecer um processo capaz de gerar um contraponto às informações oficiais. Para tanto, foi estabelecida uma parceria entre as organizações Justiça nos Trilhos (JnT), Instituto de Políticas Alternativas para o Cone Sul (PACS) e Fundação Oswaldo Cruz (Fiocruz/RJ), que culminou na construção e implementação de uma proposta de monitoramento ambiental participativo, de base comunitária, das concentrações de MP na atmosfera. Esse processo, objeto de análise deste artigo, foi denominado de vigilância popular ambiental (VPA).

A noção de vigilância popular aqui destacada se inspira em princípios e práticas de pesquisa participativa adotadas no campo da saúde coletiva, tais como: pesquisa-ação ${ }^{7}$, epidemiologia popular $^{8}$ e pesquisa participativa de base comunitária ${ }^{9,10}$. Essas abordagens ressaltam o protago- 
nismo dos sujeitos em situação de vulnerabilidade (ou vulnerabilizados pela poluição industrial imposta) na identificação e compreensão das causas e consequências dos problemas que os afligem, com o intuito de, conforme suas demandas, propor soluções capazes de transformar as realidades em que se encontram. Portanto, além de garantir um contraponto às informações oficiais (geradas pelas empresas poluidoras ou pelas agências de fiscalização competentes), a importância do estabelecimento de parcerias entre comunidades em situações de injustiça ambiental e pesquisadores acadêmicos consiste em promover a produção contextualizada de conhecimentos sobre os riscos e impactos ambientais, ação importante para visibilizar e apoiar as lutas para superar as desigualdades ambientais em questão ${ }^{3,6}$.

A poluição do ar é reconhecida como um problema de saúde pública, responsável por uma gama crescente de efeitos para a saúde que são bem documentados por extensos esforços de pesquisa realizados em muitas regiões do mundo ${ }^{11}$.

O nível de poluição atmosférica é avaliado pela quantidade de substâncias poluentes presentes no ar. A medição sistemática da qualidade do ar é restrita a um número de poluentes, definidos em função de sua importância e dos recursos disponíveis para seu acompanhamento, e entre eles está o $\mathrm{MP}^{2}$.

$\mathrm{O}$ ar poluído pode conter diversos agentes, e a fração de MP é conhecida por conter várias substâncias que podem iniciar a formação de tumores por meio de danos genéticos e mutações. Há evidência suficiente em seres humanos para a carcinogenicidade (Grupo 1/IARC) da poluição do ar e da exposição a MP. Esse tipo de exposição provoca câncer de pulmão ${ }^{12}$.

Este estudo analisou um tipo específico de poluente do ar, o MP, principalmente o de 2,5 micrômetros - $\mu \mathrm{m}\left(\mathrm{MP}_{2,5}\right)$, as chamadas partículas finas ou respiráveis ${ }^{13}$. Esse tipo de MP tem grande capacidade de penetração no sistema respiratório, gerando maiores riscos à saúde humana, e é um dos elementos presentes no tipo de emissão atmosférica gerada por atividades antropogênicas, no caso abordado neste artigo, a atividade siderúrgica $^{14,15}$.

Ao longo dos últimos anos, houve um aumento substancial nas descobertas de que a poluição atmosférica por MP não só exerce um grande impacto à saúde como também está associada a um maior número de doenças. Os da- dos sugerem que os efeitos negativos na saúde podem ocorrer mesmo em baixos níveis ${ }^{16,17}$. O efeito final da poluição do ar na saúde pública é provocar a morte prematura. Estudos recentes de longo prazo mostram associações consistentes e significativas entre MP e mortalidade em níveis bem abaixo do atual nível anual de referência da qualidade do ar da Organização Mundial de Saúde (OMS) para $\mathrm{MP}_{2,5}{ }^{11}$.

A OMS publicou em 2006, à luz dos conhecimentos da época, uma revisão dos valores-guia para os poluentes atmosféricos, visando a proteção da saúde da população ${ }^{15}$. Esses valores são usados até hoje, sendo o parâmetro usado como referência pela legislação brasileira atual ${ }^{18}$. Nessa publicação, os padrões de qualidade do ar variam de acordo com a abordagem adotada para balancear riscos à saúde, viabilidade técnica, considerações econômicas e vários outros fatores políticos e sociais, que por sua vez dependem, entre outros aspectos, do nível de desenvolvimento e da capacidade do Estado de gerenciar a qualidade do ar.

Em trabalhos mais recentes, pesquisadores analisam problemas ambientais e de saúde relacionados à expansão da mineração e da siderurgia no país, e a consequente poluição atmosférica, além de outras atividades econômicas com potencial poluidor. Tais atividades envolvem o uso intensivo de recursos naturais e inúmeros impactos ambientais e sanitários sobre os territórios e populações onde são realizadas ${ }^{19,20}$.

O crescimento da exploração de minério de ferro e das atividades siderúrgicas (produção de ferro gusa e de aço) tem agravado os problemas e conflitos ambientais no Brasil. Isso vem fazendo com que diversas entidades, movimentos sociais e populações atingidas se mobilizem em torno desses conflitos e demandem estudos e assessoria técnica para melhor compreender a questão e atuar contra os problemas gerados, relacionados a saúde, ambiente e ameaças aos direitos humanos fundamentais ${ }^{20}$.

Assim, o objetivo deste trabalho foi analisar as experiências de vigilância popular ambiental desenvolvidas em duas localidades impactadas por empreendimentos do setor siderúrgico: Santa Cruz, no município do Rio de Janeiro (RJ), e Piquiá de Baixo, no município de Açailândia (MA). Em ambos os casos foram realizadas atividades de monitoramento ambiental comunitário (MAC) dos níveis de $\mathrm{MP}_{2,5}$ na atmosfera entre novembro de 2016 e julho de 2017. 


\section{Metodologia}

\section{Locais de estudo}

A atividade siderúrgica chegou a Santa Cruz no ano de 2010 com a Thyssenkrupp - Companhia Siderúrgica do Atlântico (TKCSA), localizada na avenida João XXIII, área de conjuntos habitacionais, agricultura e pesca do bairro de Santa Cruz, na zona oeste da cidade (PACS, 2012), um bairro que já tinha uma área industrial importante, como as de produção de tintas e solventes, fabricação de couro, impressão, fabricação de aço estrutural e metalurgia ${ }^{21}$. Em 2017, a TKCSA foi vendida para a Ternium (nome atual), empresa que hoje administra a siderúrgica. A área está situada entre os canais São Francisco Xavier e Guandu, a Av. João XXIII e a Baía de Sepetiba.

Piquiá de Baixo é uma comunidade localizada na cidade de Açailândia, situada no oeste do Maranhão, fazendo divisa com o Pará. A população do município é estimada em 112.000 habitantes ${ }^{22}$. Piquiá de Baixo é um dos primeiros bairros de Açailândia, formado nos anos 1970. Ao final dos anos 1980, instalaram-se nesse bairro cinco siderúrgicas, operando com 14 altos-fornos. Atualmente, Piquiá abriga cerca de 320 famílias e a população é estimada em 1.200 pessoas $^{23}$.

\section{Oficina de capacitação do(a)s jovens}

O processo de capacitação da equipe de jovens de Santa Cruz e Piquiá foi o primeiro passo, e etapa muito importante, para a formação em VPA. Em outubro de 2016 foi realizada em Piquiá, com jovens das duas comunidades, a oficina "Vigilância popular em saúde e ambiente: monitoramento comunitário de materiais particulados na atmosfera".

Nessa oficina foram apresentados e discutidos conceitos relacionados a: saúde e ambiente; poluição atmosférica e impactos à saúde; princípios de coleta ambiental e tratamento dos resultados. Optou-se por focar as questões ambientais e seus desdobramentos sobre a saúde, de modo a envolver e sensibilizar os jovens quanto à importância das ações propostas na realidade que os cerca, refletindo inclusive o que há de mais recente no campo de estudos sobre exposição ao MP e efeitos na saúde humana ${ }^{17}$.

\section{Monitoramento ambiental}

Para a realização do MAC foi utilizado o equipamento monitor de qualidade do ar da marca Dylos, modelo DC 1700 (Dylos Corporation, CA/EUA). Foram adquiridas duas unidades desse monitor, a partir de recursos captados pelo PACS, e a escolha do modelo de equipamento se deu pelo baixo custo e por sua fácil operação.

Os parâmetros nacionais e internacionais, adotados neste trabalho, para avaliação da qualidade do ar utilizam como unidade de medição o micrograma por metro cúbico de ar $\left(\mu \mathrm{g} / \mathrm{m}^{3}\right)^{15,18}$ para fazer referência à concentração de MP no ar, porém o equipamento exibe apenas a contagem de partículas. Assim, de acordo com recomendação do fabricante, para fazer uma conversão para $\mathrm{MP}_{2,5}$, em $\mu \mathrm{g} / \mathrm{m}^{3}$, foi usada a diferença entre as leituras de partículas (pequenas e grandes) do equipamento, com a subsequente divisão por 100 . Dessa forma foi possível converter, de maneira aproximada, a leitura de número de partículas por volume de ar (contagem) para massa por volume de ar (concentração). A conversão habilitou um equipamento de simples operação e baixo custo para um estudo desse tipo. Ainda de acordo com fabricante, é importante ter ciência de que, como em todos os instrumentos de dispersão de luz, essa é uma aproximação, mas que tem funcionado adequadamente para avaliar a poluição do ar.

Para conferir os dados obtidos com o equipamento Dylos foram realizados testes comparativos com outro equipamento monitor de MP, o Optical Particle Sizer Spectrometer, modelo 3330 (TSI Incorporated, MN/USA). Esse equipamento é um modelo de monitor de partículas mais sensível e robusto, usado normalmente em pesquisas de avaliação quantitativa de MP no ar.

Em ambos os locais, Piquiá e Santa Cruz, o período de monitoramento da $1^{\text {a }}$ campanha foi entre novembro de 2016 a janeiro de $2017 . \mathrm{Na}^{\mathrm{a}}$ campanha, o período de monitoramento em Piquiá foi de março a maio de 2017, enquanto em Santa Cruz foi de maio a julho de 2017.

Em Piquiá os pontos de coleta foram as residências de moradores, localizadas próximas do entorno do complexo siderúrgico da região. $\mathrm{O}$ mesmo em Santa Cruz, onde os pontos de coleta também eram as residências de integrantes da equipe, todas situadas nas comunidades da avenida João XXIII, o mesmo endereço da companhia siderúrgica Ternium (Figura 1).

\section{Resultados e discussão}

Os resultados obtidos no processo de MAC realizado nas localidades de Piquiá e Santa Cruz são apresentados a seguir. 


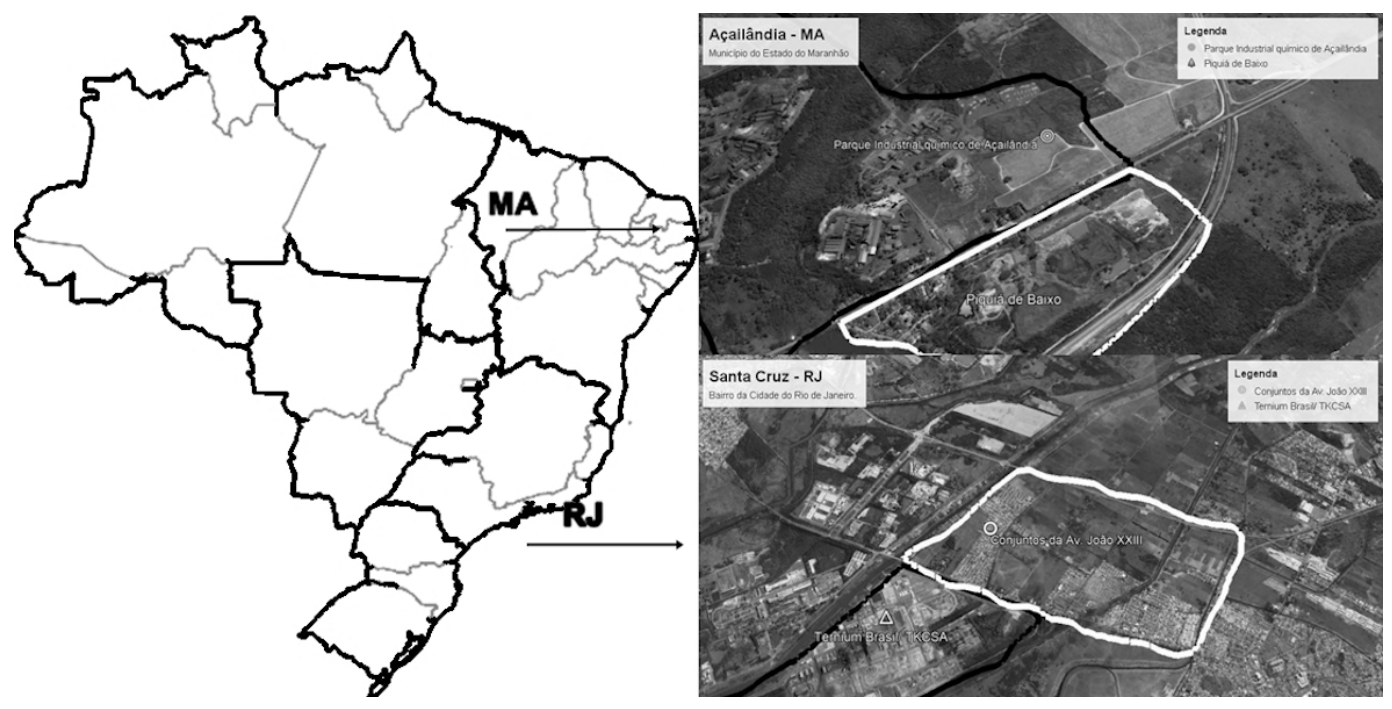

Figura 1. Mapa do Brasil ${ }^{24}$ destacando os dois estados e as localidades onde o trabalho foi realizado: Piquiá de Baixo/MA e Santa Cruz/RJ.

Fonte: Elaborado pelos autores, com auxílio do programa Google Earth.

As coletas foram feitas nos períodos da manhã, tarde, noite e madrugada, porém os resultados para calcular as médias mensais foram considerados como representativos de único período (média ponderada do dia de coleta), pois, em avaliação prévia, foi verificado que os níveis de poluição no decorrer do dia não tiveram grande variação, com algumas exceções. A Tabela 1 apresenta as médias mensais dos resultados obtidos nas duas campanhas de MAC realizadas em Piquiá e Santa Cruz.

De acordo com a Tabela 1, observa-se que os resultados (médias mensais) encontrados na $1^{\text {a }}$ campanha (2016-2017) de MAC em Piquiá são elevados em todos os meses, pois ultrapassam o limite anual recomendado pela OMS, de $10 \mu \mathrm{g} / \mathrm{m}^{3}$, nos três meses avaliados. $\mathrm{O}$ limite diário recomendado pela OMS, de $25 \mu \mathrm{g} / \mathrm{m}^{3}$, também foi ultrapassado em várias medições diárias de novembro de 2016, e algumas vezes nos meses seguintes.

Observa-se ainda comportamento parecido na $2^{\text {a }}$ campanha de Piquiá (2017), onde o limite anual é ultrapassado em todos os meses avaliados e o limite diário de $25 \mu \mathrm{g} / \mathrm{m}^{3}$ foi ultrapassado em alguns dias de março e abril e com maior frequência em maio de 2017. Isso demonstra que os níveis de poluição do ar em Piquiá de Baixo são preocupantes.
Tabela 1. Médias mensais obtidas nas duas campanhas de monitoramento ambiental comunitário realizadas em Piquiá de Baixo e Santa Cruz.

\begin{tabular}{|c|c|c|c|}
\hline \multicolumn{4}{|c|}{ Piquiá de Baixo } \\
\hline & \multicolumn{3}{|c|}{$\begin{array}{l}\text { Média de concentração } \\
\text { de } \mathrm{MP}_{2,5}\left(\mu \mathrm{g} / \mathrm{m}^{3}\right)\end{array}$} \\
\hline Período de coleta & Novembro & Dezembro & Janeiro \\
\hline $\begin{array}{l}\text { 1a campanha } \\
\text { (2016-2017) }\end{array}$ & 32,2 & 18,4 & 16,3 \\
\hline Período de coleta & Março & Abril & Maio \\
\hline $\begin{array}{l}2^{\text {a }} \text { campanha } \\
(2017)\end{array}$ & 16,0 & 18,2 & 22,3 \\
\hline \multicolumn{4}{|c|}{ Santa Cruz } \\
\hline & \multicolumn{3}{|c|}{$\begin{array}{l}\text { Média de concentração } \\
\text { de } \mathrm{MP}_{2,5}\left(\mu \mathrm{g} / \mathrm{m}^{3}\right)\end{array}$} \\
\hline Período de coleta & Novembro & Dezembro & Janeiro \\
\hline $\begin{array}{l}\text { 1a campanha } \\
(2016-2017)\end{array}$ & 15,0 & 17,6 & 10,1 \\
\hline Período de coleta & Maio & Junho & Julho \\
\hline $\begin{array}{l}2^{\text {a }} \text { campanha } \\
(2017)\end{array}$ & 30,2 & 30,8 & 34,0 \\
\hline
\end{tabular}

Fonte: Elaborado pelos autores.

Ainda de acordo com a Tabela 1, observa-se que os resultados encontrados na $1^{\text {a }}$ campanha (2016-2017) de MAC em Santa Cruz foram mais 
altos em novembro e dezembro de 2016 do que o limite anual recomendado pela OMS e ficaram dentro da média recomendada em janeiro de 2017. O limite diário recomendado pela OMS não foi ultrapassado, exceto em dois dias de dezembro de 2016, ao longo dos três meses da $1^{\text {a }}$ campanha (2016-2017). Na 2a campanha, chama a atenção o aumento significativo nos valores de concentração média de $\mathrm{MP}_{2,5}$. $\mathrm{O}$ monitoramento executado nesse período evidencia que, ao longo dos três meses monitorados em 2017, os níveis mensais de poluição são superiores (três vezes maiores) à média anual recomendada pela OMS. Além disso, o limite diário recomendado foi ultrapassado em vários dias de monitoramento. Cabe lembrar que essa época do ano na cidade do Rio de Janeiro é caracterizada como um período predominantemente seco, o que favorece a concentração de MP na atmosfera ${ }^{21}$.

As tabelas 2 e 3 apresentam uma comparação dos resultados encontrados em Piquiá de Baixo e Santa Cruz nos dois períodos avaliados.

Na Tabela 2 observa-se que Piquiá apresenta maiores níveis de poluição do ar em relação a Santa Cruz, com uma diferença elevada (o dobro) no mês de novembro de 2016 e uma diferença menor em janeiro de 2017, enquanto em dezembro de 2016 as duas localidades apresentaram a mesma concentração média de $\mathrm{MP}_{2,5}$. Nota-se que em ambas as localidades as médias de concentração tendem a diminuir em janeiro ( $1^{\text {a }}$ campanha). Isso pode ser atribuído ao fato de

Tabela 2. Comparação das médias mensais obtidas na $1^{\text {a }}$ campanha (2016-2017) do monitoramento ambiental.

\begin{tabular}{lccc}
\hline & \multicolumn{3}{c}{ Média de concentração de $\mathbf{M P}_{2,5}\left(\mu \mathrm{g} / \mathbf{m}^{3}\right)$} \\
\cline { 2 - 4 } & Novembro & Dezembro & Janeiro \\
\hline Piquiá & 32,2 & 18,4 & 16,3 \\
Santa Cruz & 15,0 & 17,6 & 10,1 \\
\hline Fonte: Elaborado pelos autores. & &
\end{tabular}

Fonte: Elaborado pelos autores.

Tabela 3. Comparação das médias mensais obtidas na 2a campanha (2017) do monitoramento ambiental.

\begin{tabular}{lccc}
\hline & \multicolumn{3}{c}{ Média de concentração de $\mathrm{MP}_{2,5}\left(\boldsymbol{\mu g} / \mathrm{m}^{3}\right)$} \\
\cline { 2 - 4 } & Março & Abril & Maio \\
\hline \multirow{2}{*}{ Piquiá } & 16,0 & 18,2 & 22,3 \\
\cline { 2 - 4 } & Maio & Junho & Julho \\
Santa Cruz & 30,2 & 30,8 & 34,0 \\
\hline Fonte: Elaborado pelos autores. & &
\end{tabular}

esse ser um mês mais chuvoso, o que precipita a poluição em suspensão no ar, o chamado efeito wash-out ${ }^{25}$, como é o caso do MP. Em termos climáticos, as duas regiões apresentam um verão chuvoso, ou seja, as chuvas entre os meses de dezembro e fevereiro se intensificam, contribuindo para uma diminuição nos níveis de poluição no período. No estudo de Mariani e Mello ${ }^{26}$, realizado na região metropolitana do Rio de Janeiro/RJ, as autoras também reportaram diferentes resultados para médias diárias de $\mathrm{MP}_{2,5}$ nas estações seca e chuvosa $\left(24,4\right.$ e $13,4 \mu \mathrm{g} / \mathrm{m}^{3}$, respectivamente), evidenciando a importância desse fator nos níveis de MP.

Na Tabela 3 verifica-se que em Piquiá as médias cresceram discretamente no período de março a maio de 2017, fato possivelmente associado à redução das chuvas na região, enquanto no Rio de Janeiro o período de maio a julho é mais seco, de maior estabilidade meteorológica, gerando médias bem próximas, e também bastante elevadas, quando comparadas à média anual recomendada pela OMS $\left(10 \mu \mathrm{g} / \mathrm{m}^{3}\right)$.

As médias obtidas pelas atividades de MAC nas duas localidades foram comparadas com os limites recomendados pela OMS para qualidade do ar, que são os mesmos valores usados pela Resolução CONAMA no 491 , de $2018^{18}$, para os padrões finais de qualidade do ar adotados na legislação brasileira nos dias de hoje, mostrando a atualidade e importância dos limites recomendados pela OMS.

É importante destacar que o limite anual da OMS foi usado como parâmetro para a avaliação comparativa das médias mensais obtidas neste estudo, uma vez que o monitoramento realizado não tem dados do ano completo para calcular uma média anual.

Para verificar a exatidão dos dados obtidos com o equipamento Dylos, foram feitos paralelamente testes de coleta de ar usando o monitor TSI, modelo 3330. As coletas com os dois equipamentos foram executadas em ambientes indoor $\mathrm{e}$ outdoor, ao mesmo tempo e nas mesmas condições, e os dados obtidos foram comparados. Em geral os dados gerados pelo equipamento TSI eram levemente maiores do que os mesmos dados de coleta do Dylos. Essa observação era esperada, uma vez que o equipamento da TSI é um monitor de ar mais sensível e exato. Mesmo diferentes, os valores eram bem próximos em termos de concentração. Isso mostra que os resultados obtidos pelo equipamento Dylos são confiáveis para esse tipo de monitoramento. Outra observação importante nos testes comparativos é que, 
se os resultados do Dylos são ligeiramente menores quando comparados a um monitor de ar mais sensível, os valores aqui discutidos poderiam ser maiores, gerando uma preocupação ainda maior com os resultados auferidos em nosso estudo, que, mesmo com essa leve tendência de apresentar valores de concentração menores que o valor real, ainda assim apresentam médias diárias e mensais de $\mathrm{MP}_{2,5}$ acima do recomendado pela OMS em diversas ocasiões.

Em outros trabalhos realizados em anos anteriores na mesma região da avenida João XXIII em Santa Cruz, foram encontrados resultados semelhantes ao deste estudo ${ }^{21,25}$. Nesses trabalhos, os valores recomendados pela OMS para $\mathrm{MP}_{2,5}$, sejam os limites diários ou anuais, foram excedidos. Não foram encontrados artigos que fizeram esse tipo de avaliação ambiental em Piquiá, somente em Santa Cruz.

No trabalho conduzido por Mateus e colaboradores $^{25}$, os autores acharam uma média anual de $\mathrm{MP}_{2,5}$ de $12,4 \pm 8,8$ e $12,1 \pm 8,5 \mu \mathrm{g} / \mathrm{m}^{3}$ nas regiões do CIEP João XXIII e do conjunto Alvorada, respectivamente, ambas em Santa Cruz. Os valores são referentes a coletas que ocorreram entre agosto de 2010 e julho 2011, o ano em que começou a operação da antiga TKCSA, agora Ternium. Os autores chamam a atenção para os valores encontrados já nessa época, que estavam acima do valor anual recomendado pela OMS, lembrando que a operação da indústria siderúrgica na região estava apenas no início. Os resultados de MP encontrados por Mateus e colaboradores ${ }^{25}$ mostram que nosso estudo está na mesma faixa de grandeza e com resultados relativamente próximos para alguns meses.

No estudo conduzido por Rodríguez-Cotto e colaboradores $^{21}$, os autores analisaram o $\mathrm{MP}_{10}$ e o $\mathrm{MP}_{2,5}$ na mesma região de Santa Cruz e seus efeitos sobre o sistema imune (respostas inflamatórias e tóxicas). As coletas ambientais ocorreram em 2010 e a média anual de $\mathrm{MP}_{2,5}$ também foi de $12 \mu \mathrm{g} / \mathrm{m}^{3}$, assim como no estudo de Mateus e colaboradores ${ }^{25}$ no mesmo local e época. Já os valores encontrados para a média diária de $\mathrm{MP}_{2,5}$ foram: 43,4 e $29,4 \mu \mathrm{g} / \mathrm{m}^{3}$ nos meses de agosto e setembro de 2010, respectivamente, para a região do CIEP João XXIII, e 42,8 e $21,1 \mu \mathrm{g} / \mathrm{m}^{3}$ para a região do conjunto Alvorada no mesmo período. Isso mostra valores que excedem o limite máximo diário de $25 \mu \mathrm{g} / \mathrm{m}^{3}$ recomendado pela OMS. Essas concentrações encontradas por RodríguezCotto e colaboradores ${ }^{21}$ para as médias diárias foram parecidas com nossos resultados de médias mensais de maio a julho de 2017.
No "Relatório sobre qualidade do ar do estado do Rio de Janeiro, ano-base 2015”, avaliação realizada pelo Instituto Estadual do Ambiente do Rio de Janeiro - INEA/RJ, são apresentados dados do monitoramento ambiental para poluição atmosférica em todo o estado do RJ obtidos pelas estações espalhadas por várias localidades ${ }^{27}$. O monitoramento de $\mathrm{MP}_{2,5}$ no Rio de Janeiro contou com 17 estações semiautomáticas em 2015, sendo duas dela em Santa Cruz, uma no conjunto Alvorada e outra na região da avenida João XXIII, mesmas localidades de nosso estudo. Segundo o relatório sobre os resultados do monitoramento de $\mathrm{MP}_{2,5}$ em 2015, algumas estações apresentariam violações caso fossem adotados os valores utilizados como os padrões diário e anual da Agência de Proteção Ambiental dos EUA - EPA $\left(35 \mu \mathrm{g} / \mathrm{m}^{3}\right.$ e $12 \mu \mathrm{g} / \mathrm{m}^{3}$, respectivamente). De acordo com os dados apresentados, observa-se valores elevados em Santa Cruz: no padrão diário, o conjunto Alvorada ultrapassou $35 \mu \mathrm{g} / \mathrm{m}^{3}$ (excede o limite da OMS, de $25 \mu \mathrm{g} / \mathrm{m}^{3}$ ), e o ponto João XXIII apresentou média de $25 \mu \mathrm{g} / \mathrm{m}^{3}$ (no limite da OMS). Já para o padrão anual, ambas as localidades ficaram abaixo do padrão da EPA, porém a média obtida foi de $10 \mu \mathrm{g} / \mathrm{m}^{3}$, exatamente o limite máximo do padrão da OMS que temos discutido neste artigo.

É importante lembrar das limitações de um estudo como este que realizamos, com uma abordagem técnica diferenciada, uma vez que a metodologia usada não busca "assinaturas de fonte de exposição", ou seja, a poluição analisada pelo monitor Dylos é a poluição ambiental geral, aquela emitida por qualquer fonte, natural ou antropogênica, como atividades industriais (de qualquer tipo), queima de combustíveis fósseis, queimadas etc., todas elas fontes de MP (entre eles o $\mathrm{MP}_{2,5}$ ), entre outros tipos de poluentes atmosféricos ${ }^{28,29}$.

A forma de quantificar a poluição atmosférica que usamos também tem suas limitações, pois utilizamos equipamento monitor simples, que analisa a contagem de partículas, e depois uma conversão foi feita para obter a concentração, enquanto a avaliação ambiental mais detalhada usa diversos tipos de equipamentos, com precisão e exatidão adequadas para esse tipo de trabalho, o que inclui determinação direta da concentração de MP $\left(\mathrm{em} \mu \mathrm{g} / \mathrm{m}^{3}\right)$ e análises laboratoriais de suporte, para a qualificação e quantificação dos tipos de poluentes coletados, conforme pode ser observado em outros estudos de avaliação da poluição atmosférica em áreas com atividade siderúrgica $^{21,25,30}$.

Ainda cabe lembrar da limitação dos locais avaliados, pois neste tipo de vigilância popular, 
os pontos de coleta foram os pontos acessíveis aos jovens, prioritariamente suas próprias residências, enquanto em um estudo mais detalhado de poluição ambiental a área de coleta é previamente avaliada, buscando definir os pontos mais adequados, julgando a dispersão dos poluentes e a representatividade (geográfica e numérica) da área de estudo e dos pontos de coleta, respectivamente ${ }^{28}$.

Mesmo com as limitações apresentadas, a riqueza de informações e formação que esse tipo de abordagem participativa traz torna muito importante a continuidade de trabalhos nesses moldes. Um desdobramento muito importante é a experiência bem-sucedida de capacitar moradores de comunidades que sofrem com a poluição atmosférica e que querem fazer esse tipo de MAC para gerar dados próprios.

Os resultados aqui apresentados e discutidos mostram a importância de um programa de VPA, por meio do MAC, utilizando um equipamento monitor simples e barato. $\mathrm{O}$ baixo custo de aquisição, aliado à facilidade de ser operado por pessoas não-especializadas, faz com que esses equipamentos sejam de grande valia em trabalhos desse tipo, em que a comunidade pode ser o próprio gerador de dados para avaliação de cenários de poluição atmosférica, mas que de forma alguma devem ser usados no lugar de dados oficiais de monitoramento ambiental realizado pelos órgãos competentes.

Com o objetivo de verificar o que tem sido feito no campo de abordagem participativa para enfrentar problemas ambientais com envolvimento da comunidade na geração de dados técnicos, foram buscados trabalhos análogos. Alguns estudos interessantes, de âmbito nacional, foram encontrados, porém voltados para o monitoramento de águas ${ }^{31-33}$.

No trabalho de Hermes e colaboradores ${ }^{32}$ foi realizado um processo participativo/comunitário de monitoramento da qualidade da água. Apesar do compartimento ambiental avaliado ter sido diferente do analisado no presente artigo, os autores ressaltam que todos, não apenas os profissionais especializados, podem estudar e entender o local onde moram, refletir criticamente sobre os problemas ambientais vivenciados e participar ativamente de tomadas de decisão junto à comunidade e ao poder público local. Essa ideia apresentada pelos autores vai ao encontro da proposta de VPA, uma vez que busca envolver a comunidade atingida como protagonista na execução das atividades de produção e avaliação de dados sobre os problemas ambientais aos quais está submetida. Tal iniciativa visa garantir uma participação mais ativa e qualificada nas discussões públicas que podem embasar as decisões referentes à gestão dos problemas enfrentados.

No estudo de Figueirêdo e colaboradores ${ }^{33}$ também é abordado o monitoramento comunitário da qualidade da água como uma ferramenta para a gestão participativa dos recursos hídricos. O estudo avaliou os benefícios oriundos da adoção do modelo de monitoramento comunitário dos Vigilantes da Água Global (Global Water Watch) no semiárido brasileiro. Os autores destacam que os resultados obtidos com a implantação do modelo reforçam a importância do "aprender fazendo" na mudança de atitude e na criação de uma consciência política, atributos essenciais à participação ativa da sociedade na gestão das águas. O "aprender fazendo" que se destaca foi exatamente o que se constatou na experiência desenvolvida em parceria com os jovens durante a oficina de formação em VPA, e depois no encontro para analisar os dados obtidos. Foi possível observar claramente o aprendizado dos jovens após o período de coleta e a continuidade desse processo de formação.

No trabalho de Buss ${ }^{31}$ sobre o desenvolvimento de protocolos de bioavaliação rápida da qualidade da água de rios e seu uso por agentes comunitários na gestão de recursos hídricos, o autor fala sobre importância da participação no monitoramento ambiental pela comunidade, fazendo com que informações sejam geradas localmente, com autonomia do grupo de voluntários, indicando empoderamento das comunidades, que passam a contar com informações importantes para pleitear seu papel nos espaços de decisão acerca dos planos de recursos hídricos.

Esses estudos corroboram a importância da abordagem participativa como ferramenta de mudança nas comunidades envolvidas. Mesmo que os estudos citados tenham trabalhado com monitoramento ambiental de águas, eles têm um paralelo muito rico com nosso trabalho de monitoramento do ar.

Outros estudos de monitoramento ambiental com abordagem participativa foram encontrados, porém reportavam o uso de aplicativos, telefones celulares e "sensores inteligentes" para fazer o monitoramento ambiental de poluição atmosférica e gerar dados para uma platafor$\mathrm{ma}^{34-36}$. Esse tipo de abordagem é muito promissora e pode ser pensada para futuros trabalhos no Brasil.

Com relação às questões sociais envolvidas no VPA em contextos de atividade industrial, não 
bastassem os impactos à saúde e ao ambiente provenientes das indústrias siderúrgicas, há outros impactos que se configuram como violações de direitos humanos ainda mais graves e sistemáticas quando a indústria poluente encontra no Estado um ente que não fiscaliza e regula devidamente suas atividades extrativas e industriais. Nesses casos, em que se configura um quadro de fragilidade, ausência ou permissividade do Estado, as autoridades locais estabelecem com o empreendimento econômico uma relação de dependência e, muitas vezes, até de subserviência ${ }^{23}$. Esse cenário apresentado tende a agravar ainda mais os riscos à saúde das comunidades afetadas e o ambiente.

Um resultado não apresentado em números, porém muito importante de se discutir, tem relação com a formação dos jovens na oficina realizada no início das atividades de VPA. A percepção dos mesmos sobre o problema que os cerca, ação aqui estimulada pelo monitoramento ambiental comunitário, e a compreensão acerca dos impactos ambientais e seus efeitos para a saúde trouxeram uma outra perspectiva sobre essa realidade. Essa nova interpretação dos problemas locais, com visão crítica quanto ao processo e aos dados apresentados pelas indústrias, impele os jovens a tentar buscar informações sobre o que acontece em suas comunidades. Esse primeiro passo já é um grande resultado, e um enorme ganho, em termos de formação de jovens na área de saúde e ambiente.

\section{Conclusão}

Cada vez mais a poluição do ar tem gerado grandes preocupações por conta dos riscos à saúde humana, entre os quais o câncer.

Mesmo utilizando um equipamento monitor de qualidade do ar de simples manuseio e baixo custo, resultados expressivos podem ser obtidos, o que é de grande valia para ações de VPA.
As médias mensais dos níveis de poluição do ar, avaliados pela concentração de $\mathrm{MP}_{2,5}$, em Piquiá de Baixo (Açailândia/MA) e em Santa Cruz (Rio de Janeiro/RJ) são elevadas, a depender da época avaliada, pois ultrapassam a média anual recomendada pela OMS, de $10 \mu \mathrm{g} / \mathrm{m}^{3}$, e por várias vezes superam a média diária recomendada, de $25 \mu \mathrm{g} / \mathrm{m}^{3}$. Esses resultados merecem atenção, devido aos potenciais efeitos à saúde causados por esse tipo de exposição, principalmente na parcela mais sensível da população (crianças e idosos).

Mesmo com limitações técnicas inerentes, as atividades de MAC se mostraram uma importante ação em termos de vigilância ambiental, pois foram executadas a partir da participação ativa, do protagonismo dos sujeitos locais atingidos. Assim, contrapõe-se à ideia de que o fazer científico deve se concentrar na esfera acadêmica, nas universidades. A experiência da VPA mostra que o conhecimento científico pode ser popularizado e apreendido por agentes de diferentes áreas e distintas particularidades, assim como para meios distintos, como neste caso, que desempenhou o papel de contra hegemonia a um discurso mercadológico, desenvolvimentista e que favorece processos geradores de injustiça ambiental

A capacitação de jovens dos locais estudados permitiu a inserção deles no processo de MAC, além de contribuir para a formação de um olhar crítico sobre questões relativas à saúde e ao ambiente. Assim, destacamos não só o resultado obtido com os dados coletados, mas o processo de formação pelo qual passaram os jovens moradores das áreas atingidas. A experiência de VPA propiciou a transformação de atores locais em agentes de mudança e multiplicadores de conhecimento, com influência ampliada na região impactada e capazes de participar de maneira mais qualificada, do ponto de vista técnico, dos processos decisórios públicos a respeito da gestão ambiental local. 


\section{Colaboradores}

Todos os autores trabalharam na concepção do estudo, análise e interpretação dos dados, na redação do artigo e sua revisão crítica, assim como na aprovação da versão final a ser publicada.

\section{Agradecimentos}

Este artigo é fruto da colaboração de diversas pessoas. Agrademos aos jovens de Piquiá e Santa Cruz, responsáveis pelas coletas do MAC, e às equipes do PACS e JnT pelo apoio à realização dessa iniciativa.

\section{Referências}

1. Souza RC. Análise dos impactos de emissões atmosféricas locais da indústria siderúrgica: um estudo de caso no Rio de Janeiro [dissertação]. Rio de Janeiro: Universidade Federal do Rio de Janeiro; 2013.

2. Vallero DA. Fundamentals of air pollution. Waltham/ San Diego/London/Oxford: Academic Press; 2014.

3. Porto MF, Finamore R, Rocha D. Saúde como dignidade: riscos, saúde e mobilizações por justiça ambiental. Congresso Brasileiro de Saúde Coletiva, Goiânia; 2015.

4. Acselrad H. Conflitos ambientais no Brasil. Rio de Janeiro: Relume Dumará/Fundação Heinrich Böll; 2004

5. Bronz D. Nos bastidores do licenciamento ambiental: uma etnografia das práticas empresariais em grandes empreendimentos. Rio de Janeiro: Contra Capa; 2016.

6. Porto MF, Finamore R. Riscos, saúde e justiça ambiental: o protagonismo das populações atingidas na produção de conhecimento. Cien Saude Colet 2012; 17(6):1493-1501.

7. Thiollent M. Metodologia da pesquisa-ação. São Paulo: Cortez; 2009.

8. Brown P. Popular Epidemiology and toxic waste contamination: lay and professional ways of knowing. $J$ Health Soc Behav 1992; 33(3):267-281.

9. Leung MW, Yen IH, Minkler M. Community based participatory research: a promising approach for increasing epidemiology's relevance in the 21 st century. Int J Epidemiol 2004; 33(3):499-506.

10. Haley E. Methods to help communities investigate environmental health issues. Pimatisiwin A J Aborig Indig Community Heal 2005; 3(1):33-58.

11. Kelly FJ, Fussell JC. Air pollution and public health: emerging hazards and improved understanding of risk. Environ Geochem Health 2015; 37(4):631-649.

12. International Agency for Cancer Research (IARC). Outdoor air pollution. IARC monographs on the evaluation of carcinogenic risks to humans v. 109; Lyon: IARC; 2016.

13. Oliveira BFA, Ignotti E, Hacon SS. A systematic review of the physical and chemical characteristics of pollutants from biomass burning and combustion of fossil fuels and health effects in Brazil. Cad Saude Publica 2011; 27(9):1678-1698.

14. Colbeck I, Lazaridis M. Aerosols and environmental pollution. Naturwissenschaften 2010; 97(2):117-131.

15. World Health Organization (WHO). Air quality guidelines global update 2005: particulate matter, ozone, nitrogen dioxide and sulfur dioxide. Copenhagen: WHO; 2006.

16. World Health Organization (WHO). Health effects of particulate matter - Policy implications for countries in eastern Europe, Caucasus and central Asia. Copenhagen: WHO; 2013.

17. World Health Organization (WHO). WHO Expert Consultation: Available evidence for the future update of the WHO Global Air Quality Guidelines (AQGs). Copenhagen: WHO; 2016.

18. Conselho Nacional do Meio Ambiente (CONAMA). Resolução n 491 , de 19 de novembro de 2018. Dispõe sobre padrões de qualidade do ar. Diário Oficial da União 2018; 20 nov. 
19. Porto MF, Milanez B. Eixos de desenvolvimento econômico e geração de conflitos socioambientais no Brasil: desafios para a sustentabilidade e a justiça ambiental. Cien Saude Colet 2009; 14(6):1983-1994.

20. Porto MFS, Menezes M. Injustiça ambiental e avaliação da poluição decorrente da produção de ferro gusa na comunidade de Piquiá de Baixo, Açailândia, Maranhão - relatório preliminar de cooperação. Rio de Janeiro: ESNP/Fiocruz; 2014.

21. Rodríguez-Cotto RI, Ortiz-Martínez MG, Rivera-Ramírez E, Mateus VL, Amaral BS, Jiménez-Vélez BD, Gioda A. Particle pollution in Rio de Janeiro, Brazil: increase and decrease of pro-inflammatory cytokines IL-6 and IL-8 in human lung cells. Environ Pollut 2014; 194:112-120.

22. Instituto Brasileiro de Geografia e Estatística (IBGE). Açailândia [Internet]. [acesso 2019 Fev 5]. Disponível em: https://www.ibge.gov.br/cidades-e-estados/ma/ acailandia.html

23. Federação Internacional dos Direitos Humanos (FIDH). Brasil: quanto valem os direitos humanos? Os impactos sobre os direitos humanos relacionados à indústria da mineração e da siderurgia em Açailândia; 2011.

24. Instituto Brasileiro de Geografia e Estatística (IBGE). Portal de mapas do IBGE. [documento da Internet]; 2011 [acessado 2019 maio 03]. Disponível em: ftp:// geoftp.ibge.gov.br/produtos_educacionais/mapas_ mudos/mapas_do_brasil/mapas_nacionais/brasil.pdf

25. Mateus VL, Monteiro ILG, Rocha RCC, Saint'Pierre TD, Gioda A. Study of the chemical composition of particulate matter from the Rio de Janeiro metropolitan region, Brazil, by inductively coupled plasma -mass spectrometry and optical emission spectrometry. Spectrochim Acta Part B 2013; 86:131-136.

26. Mariani RL, Mello WZ. $\mathrm{PM}_{2.5-10}, \mathrm{PM}_{2.5}$ and associated water-soluble inorganic species at a coastal urban site in the metropolitan region of Rio de Janeiro. Atmos Environ 2007; 41(13):2887-2892.

27. Instituto Estadual do Ambiente (INEA). Relatório sobre qualidade do ar do estado do Rio de Janeiro, ano-base 2015; 2016. [documento na Internet]. [acesso 2019 Abr 27]. Disponível em: http://www.inea.rj.gov.br/cs/ groups/public/@inter_dimfis_gear/documents/document/zwew/mtmx/ edisp/inea0131852.pdf

28. Karagulian F, Belis CA, Dora CFC, Prüss-Ustün AM, Bonjour S, Adair-Rohani H, Amann M. Contributions to cities' ambient particulate matter (PM): a systematic review of local source contributions at global level. Atmos Environ 2015; 120:475-483.

29. Kim K-H, Kabir E, Kabir S. A review on the human health impact of airborne particulate matter. Environ Int 2015; 74:136-143.

30. Quiterio SL, Silva CRS, Arbilla G, Escaleira V. Metals in airborne particulate matter in the industrial district of Santa Cruz, Rio de Janeiro, in an annual period. Atmos Environ 2004; 38(2):321-331.

31. Buss DF. Desenvolvimento de protocolos de bioavaliação rápida da qualidade da água de rios e seu uso por agentes comunitários na gestão de recursos hídricos [tese]. Rio de Janeiro: Fiocruz; 2008.
32. Hermes LC, Fay EF, Buschinelli CCA, Silva AS, Silva EFF. Participação comunitária em monitoramento da qualidade da água. Circular Técnica, n. 8. Embrapa Meio Ambiente. São Paulo: Embrapa Meio Ambiente; 2004.

33. Figueirêdo MCB, Vieira VDPPB, Mota S, Rosa MF, Araujo LDFP, Girão Ê, Ducan BL. Monitoramento comunitário da qualidade da água: uma ferramenta para a gestão participativa dos recursos hídricos no semiárido. Rega 2008; 5(1):51-60.

34. Yang Y, Li L. A smart sensor system for air quality monitoring and massive data collection. International Conference on Information and Communication Technology Convergence (ICTC); 2015. p. 147-152.

35. Yang B, Castell N, Pei J, Du Y, Gebremedhin A, Kirkevold $\varnothing$. Towards crowd-sourced air quality and physical activity monitoring by a low-cost mobile platform. Lect Notes Comput Sci 2016; 9677:451-463.

36. Jafari H, Xiangfang Li, Qian L, Chen Y. Community based sensing: a test bed for environment air quality monitoring using smartphone paired sensors. 36th IEEE Sarnoff Symposium. Newark: IEEE; 2015. p. 1217.

Artigo apresentado em 16/07/2019

Aprovado em 02/12/2019

Versão final apresentada em 04/12/2019

Editores-chefes: Romeu Gomes, Antônio Augusto Moura da Silva 
